\title{
J3.13 A SPACE-TIME MODEL FOR SEASONAL HURRICANE PREDICTION
}

\author{
Thomas H. Jagger ${ }^{1}$, Xufeng Niu, James B. Elsner \\ Florida State University, Tallahassee, Florida
}

\section{INTRODUCTION}

Hurricanes are destructive natural phenomena. On average a half of dozen or so form each year over the warm tropical and subtropical waters of the North Atlantic and track westward for thousands of kilometers. Historically, hurricanes account for a majority of the costliest weather disasters in the United States. They rival earthquakes in destructive potential and loss of life. Despite technological advances in monitoring and prediction, hurricanes retain their potential to cause severe damage and numerous deaths (Arguez and Elsner 2001). During 1998, hurricane Mitch became a grim reminder that hurricanes can quickly kill thousands of people. In the United States, population and demographic shifts toward the coast are making the problem worse as development flourishes in areas prone to hurricane strikes: the warm subtropical shorelines and islands of the Atlantic Ocean and Gulf of Mexico. Knowledge of their past occurrence, even if it is incomplete, provides clues about their future frequency and intensity that goes beyond what present numerical climate models are capable of. This understanding is important for land-use planning, emergency management, hazard mitigation, (re)insurance applications and, potentially, the long-term weather derivative market. Climatologists have been issuing seasonal hurricane activity forecasts for the North Atlantic using since 1984 (Gray, 1984; Elsner et al., 1996).

Some understanding has been achieved in solving the climate puzzle with regard to the question of where hurricanes are likely to go based on conditions a month or two in advance of the season. However, current seasonal forecast models for the entire North Atlantic basin do not incorporate spatial and temporal information. Thus, they fail to provide specific seasonal activity forecasts for different geographic regions of the hurricane basin, which includes the

\footnotetext{
${ }^{1}$ Corresponding author address: Thomas $\mathrm{H}$. Jagger, Insightful Corp., 1700 Westlake Ave N., Suite 500, Seattle, WA 98109-3044, email:tjagger@insightful.com
}

Gulf of Mexico and Caribbean Sea. This paper, an abstract of Jagger et al. (2001), introduces a new class of space-time statistical models for count data that can be used for seasonal hurricane prediction. These models incorporate both the spatial and temporal correlation observed in hurricane activity.

\section{DATA}

Hurricanes are tropical cyclones with maximum sustained winds reaching $65 \mathrm{kt}$ or greater. Hurricane positions and intensities are obtained from the besttrack records (Neumann et al., 1999), which are a compilation of the six-hourly information of all tropical cyclones back to 1886 . For this paper we use data data from year 1900 through year 2000. However, there are issues that could bias our model using the hurricane data before the use of aircraft reconnaissance in 1944. These issues are addressed in the full paper by considering the relationships between the pre and post 1944 starting and ending positions for the hurricanes in the best track data set. Although this limited comparison does not prove the reliability of the earlier years, it does provide confidence that the modeling results presented in this paper will not be overly influenced by a potential data bias.

\section{MODEL GRID}

For the spatial structure of our model we divide the North Atlantic basin into a $6^{\circ}$ by $6^{\circ}$ latitude and longitude grid. Data consists of the annual hurricane counts in the grid boxes. A tropical cyclone that records a position at hurricane intensity within the box is counted once. A hurricane that loops around and reenters the box is counted as a single hurricane. Grid choice is a compromise between sample size and resolution.

We remove grids having mostly land or historically low hurricane activity, leaving grid $S$ with 40 cells. Region $S$ with the total hurricane occurrences over the 94-yr period 1899 through 1993, are shown in Fig. 1. We use count values in the grid boxes outside region 


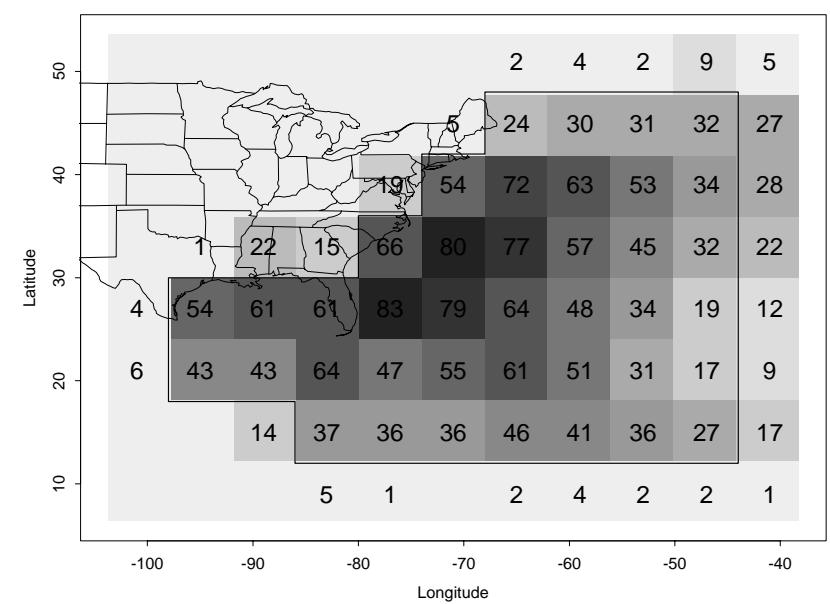

Figure 1: Grid boxes indicate the total number of hurricanes over the period 1900-93. The black line (region $S$ ) outlines the 40 grid boxes used in the model. Hurricane activity in the surrounding boxes are used as boundary conditions.

$S$ as boundary conditions for the model. However, since these are not available when creating sample forecasts, we set the boundary values to climatology.

\section{RIGHT-TRUNCATED POISSON SPACE-TIME MODEL}

Hurricane frequencies over time and space form a space-time counts process. The dependence structure of this type of data can be modeled by a conditional probability approach (Bartlett, 1968; Whittle, 1963; Besag, 1974; Gilks et al., 1996). Besag (1974) introduce conditionally specified auto-Poisson models for spatial counts data, which link observation of a Poisson process at a given location with those at its spatial neighborhoods. Since the auto-Poisson model proposed by Besag (1974) does not allow positive coupling, we consider a class of space-time auto-regression,(TPSTAR), models for hurricane activity based on the right-truncated Poisson distribution:

$$
\operatorname{Pr}(H=x)=\frac{\lambda^{x}}{x ! \sum_{w=0}^{M}\left(\lambda^{w} / w !\right)}, x=0, \ldots, M
$$

where $M$ is an upper bound for the annual number $H$ of hurricanes occurring in a given grid box during any given year.

We restrict the specification to a spatially invariant, nearest neighbor, first order autoregressive model, with predictors. The space-time neighborhood consists of the current season's north, south, east and

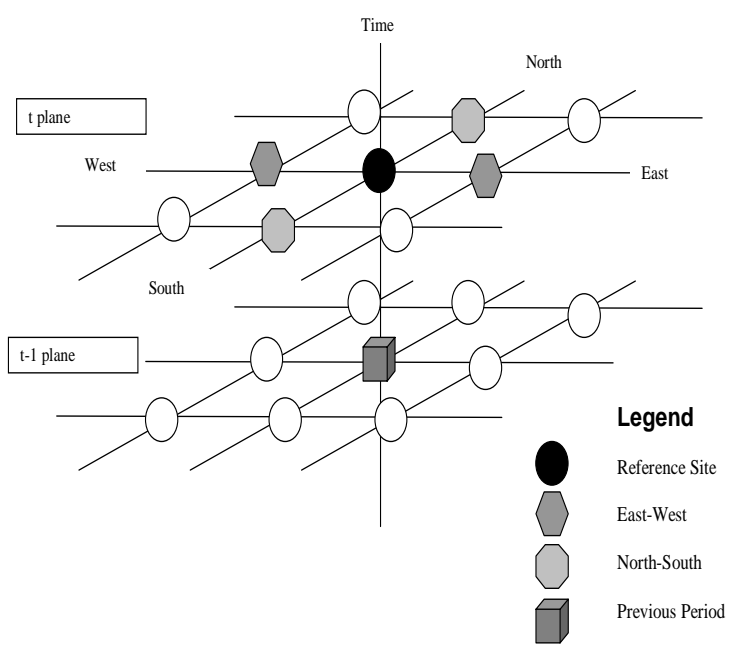

Figure 2: The space-time neighborhood of the reference grid box (site) used to model annual hurricane activity. The shaded objects indicate grid boxes used in the model. The previous period represents last year's hurricane activity in the grid box of interest.

west sites values as well as the previous seasons value as shown in Fig. 2. The TPSTAR model based on this neighborhood requires three coupling parameters $\gamma_{0 h}, \gamma_{0 v}, \gamma_{1 c}$ for the east-west, the north-south and the temporal lag-one coupling, respectively.

Each grid box $(t, i j)$ represents a $6^{\circ}$ by $6^{\circ}$ region for one year. Let the response values $H_{t, i j}$, be the number of distinct hurricanes passing into any portion of the grid $(i, j) \in S$ during year $t$. The location centers of each grid box shown in Fig. 1 have longitude given by $-101^{\circ}+6^{\circ} \cdot i$ for $i=0 \ldots 10$ and latitude given by $9^{\circ}+6^{\circ} \cdot j$ for $j=0 \ldots 7$. Region $S$ has $i=1 \ldots 9$ and $j=1 \ldots 6$.

We can describe the distribution model conditionally as:

$$
\forall i j \in S: H_{t, i j} \mid h_{\partial\{(t, i j)\}} \sim \operatorname{tpois}\left(\lambda_{t, i j}, M\right)
$$

where $h_{\partial(t, i j)}$ is the vector of five response values, $h_{t, i-1, j}, h_{t, i+1, j}, h_{t, i, j-1}, h_{t, i, j+1}$ and $h_{t-1, i, j}$, in the neighborhood of the location, $(t, i, j)$, and tpois is the righttruncated Poisson distribution with rate $\lambda_{t, i j}$ truncated at $M$.

Thus, We have the following form for the TPSTAR model of hurricane activity:

$$
\begin{aligned}
\log \left(\lambda_{t, i j}\right)= & \mathrm{C}_{i j}(t)+ \\
& \gamma_{0 h}\left(H_{t, i+1, j}+H_{t, i-1, j}\right)+ \\
& \gamma_{0 v}\left(H_{t, i, j+1}+H_{t, i, j-1}\right)+
\end{aligned}
$$




$$
\begin{array}{ll} 
& \gamma_{1 c}\left(H_{t-1, i, j}\right) \\
\text { where } \quad \mathrm{C}_{i j}(t)=\alpha_{i j}+\sum_{k=1}^{p} \mathrm{~b}_{k} z_{k}(t)
\end{array}
$$

with $\mathrm{b}_{k}$ the coefficient for the $k^{\text {th }}$ yearly covariate and $\alpha_{i j}$ the grid $i j$ offset.

We chose $M=10$ for the grid boxes, since it is larger than the maximum number of hurricanes observed in any grid box per year. The value of $M$ is small enough to observe positive coupling, yet large enough to compare the results of this model with models based on the Poisson distribution.

The model makes use of three predictors types:

1. neighborhood response values or coupling grid box frequencies, with parameters $\gamma_{0 h}, \gamma_{0 v}$, and $\gamma_{1 c}$.

2. local offsets, represented by $\alpha_{i j}$ where

$\alpha_{i j}=$ con + long $_{i}+$ lat $_{j}$ with sum constraints $\sum_{i=1}^{\lg \mathrm{N}}$ long $_{i}=0$ and $\sum_{j=1}^{\mathrm{ltN}}$ long $_{j}=0$ where con the intercept, and $\lg N$ and ItN are the number of longitude (9), and latitude (6) regions. This reduces the number of parameters associated with offsets from 40 to 14 in the present case. Since long $_{9}$ and lat ${ }_{6}$ are determined by the sum constraints, they are not reported.

3. yearly global covariates denoted by $z_{k}(t)$.

Predictors of type three represent the climate covariates as identified by previous research studies (Jagger et al., 2001). Here we consider the influence of the covariate to be the same across grid boxes, and allow the value of the covariate to vary in intensity from year to year. The number of covariates is restricted by data availability. The climate covariates used in the TPSTAR model include:

Warm \& Cold "Warm" and "Cold" refer to the state of the EI Niño-Southern Oscillation (ENSO). This factor takes three values in the model, "Warm", +1 representing an El Niño event; "Cold", -1 , representing a La Niña event or 0 for neither event.

Dakar "Dakar" refers to annual rainfall over Dakar, Senegal.

Azores "Azores" refers to annual sea level pressure (SLP) in the Azores given in millibars.

Iceland "Iceland" refers to annual SLP in Iceland given in millibars. The difference in SLP between Iceland and the Azores (Iceland - Azores) represents the North Atlantic oscillation.

\section{COMPARING MODELS}

To better examine hurricane activity as a count process over the basin, we present results from three different models, a time-series only model, a spacetime model without instantaneous coupling parameters, and a full model with coupling parameters. The full model given in (3) represents a version of TPSTAR. The first two models are fit to a Poisson generalized linear model (GLM) with dispersion. To fit the full model we try both a maximum Poisson likelihood estimator (MPLE) and a Monte Carlo maximum likelihood estimator (MCMLE).

The MPLE extends the GLM model by adding neighborhood observations as covariates to the models. If the covariate matrix is of full rank, the MPLE using the canonical link function always produces a parameter estimate. If the model is shift invariant with finite range, the parameter estimates of the MPLE are asymptotically consistent with increasing domains (Winkler 1995). However, even in the shift invariant case, the MPLE is not necessarily efficient, and does not provide standard error estimates. We use the MCMLE method for consistent and efficient parameter estimates with consistent parameter covariance estimates as described in Geyer (1994) and used by Wu (1994). The MCMLE method was extended by Jagger (2000) to handle the autoregressive coupling in the TPSTAR model.

The MCMLE method has several problems. For one, it is computationally intensive as it uses a Markov Chain Monte Carlo method for estimating the log likelihood function. For another, the method fails to converge, unless the initial parameter estimates are close to the actual parameter values. Though the MPLE is biased in our case, the convergence problem is somewhat alleviated by using the MPLE for initial parameter estimates.

For the time-series model, we analyzed annual hurricane counts over the entire region $S$ using Poisson GLM with dispersion as is used on U.S. hurricane activity in Elsner and Bossak (2001). The parameter estimates and their standard errors are shown in Table 1. We note that the time-series model is somewhat under dispersed. Dakar rainfall, Azores pressure, and the warm phase of the El Niño are significant at $\alpha=.05$, but the autoregressive coefficient is not.

For the spatial-time series model, we modified the first model by dividing the region into $6^{\circ}$ by $6^{\circ}$ grid boxes and added the latitude and longitude offsets. Using a Poisson GLM model, We find that all the factors including the autoregressive parameter are significant at $\alpha=.05$. We also note that the variance estimates using this model are three times smaller 
Table 1: Parameter estimates and statistics for the time series model. The dispersion parameter estimate is 0.924; residual deviance is 87.0 with 87 degrees of freedom. There are 94 observations, with $\sum_{t=0}^{93} H_{t+1900}=424$.

\begin{tabular}{|r|r|c|r|r||}
\hline & Value & Std. Err. & $t$-value & units \\
\hline Intercept & 198.475 & 68.996 & 2.88 & \\
$\gamma_{1 c}$ & 0.037 & 0.0194 & 1.88 & \\
Cold & 0.193 & 0.108 & 1.78 & \\
Warm & -0.302 & 0.126 & -2.38 & \\
Dakar & 0.594 & 0.250 & 2.40 & $\mathrm{yr} / \mathrm{m}$ \\
Azores & -0.149 & 0.049 & -3.05 & $\mathrm{mb}^{-1}$ \\
Iceland & -0.046 & 0.027 & -1.71 & $\mathrm{mb}^{-1}$ \\
\hline
\end{tabular}

than those for the time-series only.

The conclusions about the parameters and their variances are not entirely valid, as the GLM assumes no instantaneous coupling, i.e. the conditional distribution given the past is independent for each site. This assumption does not hold because our data consist of hurricanes that pass between adjacent grid boxes creating correlations in annual counts between adjacent boxes. This suggests adding spatial structure to the model in the form of coupling parameters.

For the final model, we added east-west, and north-south coupling. Model estimates are derived using the results of a pseudo likelihood estimator as inputs to the MCML estimator. The MCML estimator is then run iteratively with 1000 samples at each stage. Table 2 shows the final MCML estimate after four iterations. We stopped after four iterations, since the changes between iteration two and three and three and four were similar, with the root mean square change of .2 for the $t$ statistic.

Results from the full TPSTAR model indicate all couplings are positive and significant. Again, the lag-one coupling, which was not significant in the time-series model, is significant in the space-time model. This is a new finding that provides evidence for hurricane path persistence over successive years. Locations that were threatened by a hurricane one year are more likely to be threatened again in the next year. As with the second model, the climate predictors are significant.

Estimates from the MCML estimator appear to be reasonable. The model takes considers both spatial and temporal couplings. For example, the parameter estimates are the same sign, but smaller in the final model with instantaneous couplings as compared to the Poisson GLM without instantaneous couplings,
Table 2: Parameter estimates and statistics for the truncated Poisson space-time autoregressive (TPSTAR) model.

\begin{tabular}{|r||r|r|r|r|}
\hline \hline \multicolumn{1}{|c||}{} & \multicolumn{1}{|c|}{ MPLE } & \multicolumn{3}{|c|}{ MCML Estimator } \\
\cline { 2 - 5 } & Value & Value & Std. Err. & $t$-value \\
\hline$\gamma_{0 h}$ & 0.389 & 0.358 & 0.014 & 24.90 \\
$\gamma_{0 v}$ & 0.250 & 0.287 & 0.017 & 16.75 \\
$\gamma_{1 c}$ & -0.002 & 0.079 & 0.020 & 4.03 \\
intercept & 57.412 & 148.537 & 18.473 & 8.04 \\
lat1 & -0.072 & -0.010 & 0.038 & -0.27 \\
lat2 & 0.012 & 0.023 & 0.017 & 1.35 \\
lat3 & 0.039 & 0.022 & 0.013 & 1.74 \\
lat4 & 0.044 & 0.007 & 0.011 & 0.60 \\
lat5 & -0.012 & -0.047 & 0.013 & -3.62 \\
long1 & -0.197 & -0.116 & 0.076 & -1.53 \\
long2 & -0.056 & -0.003 & 0.035 & -0.09 \\
long3 & -0.047 & -0.009 & 0.020 & -0.45 \\
long4 & -0.042 & -0.009 & 0.013 & -0.68 \\
long5 & -0.024 & -0.013 & 0.010 & -1.32 \\
long6 & -0.024 & -0.032 & 0.009 & -3.73 \\
long7 & -0.018 & -0.039 & 0.008 & -4.77 \\
long8 & -0.037 & -0.055 & 0.008 & -6.79 \\
Warm & -0.124 & -0.230 & 0.038 & -6.02 \\
Cold & 0.072 & 0.192 & 0.027 & 7.15 \\
Dakar & 0.368 & 0.768 & 0.068 & 11.31 \\
Azores & -0.042 & -0.120 & 0.013 & -9.14 \\
lceland & -0.015 & -0.028 & 0.007 & -3.96 \\
\hline
\end{tabular}


or the time-series model. This makes sense, since a positive coupling causes the expected value of any statistic to be more sensitive to changes in the predictor value than would be expected in the absence of the coupling. The parameters and the estimated standard errors from the modified MCML estimator are smaller than those obtained with the first two models. This reduction in standard error might be real owing to the addition of instantaneous coupling and offsets, but it is more likely an artifact of the apparent increase in total hurricane counts from 435 in the time series model to 1676 in the TPSTAR model.

\section{MODEL SELECTION}

The backward elimination procedure is applied to the TPSTAR model to arrive at a final model of the spatial/temporal variations in seasonal hurricane This procedure makes use of the estimated changes in the Schwartz's Bayesian Information Criteria (SBC) and the Akaike Information Criteria (AIC) to determine which factors to remove from the model. We use Wald's statistic to estimate the difference in AIC and SBC between models. We minimize the AIC to find the model with the smallest predictive error, whereas we minimize the SBC to find a consistent model (Brockwell 1991). Applying backward elimination using AIC we kept all the factors in the model whereas using SBC we kept all but the latitude factor, at $\alpha=.05$. Since we are using the model primarily for forecasting, we kept the latitude factor in the full model.

\section{MODEL FIT ISSUES AND A HINDCAST}

\subsection{Strong Couplings}

Because of the strong couplings, we needed to modify the parameter estimator. We found that some of the simulations at each time period contained large predicted values for hurricane activity. Thus, we were forced to remove simulations in the case that any cell contained the maximum value, $M=10$, of the right truncated Poisson distribution. The restriction is reasonable since the maximum observed number of hurricanes for any region during any year at any of the three regions size is four. The restriction biases the estimator in that the parameters are estimated for the spatial distribution conditioned on the maximum at any location being less than ten. This restriction will decrease the observed Fisher Information, which will tend to inflate the values of the estimated standard error.

\subsection{Grid Size}

As mentioned above, the spatial grid is a compromise between sample size and resolution. We experimented with smaller grid boxes but found that the MCML estimator did not converge. In these case we lack accurate information for estimating the coupling parameters. The $6^{\circ}$ by $6^{\circ}$ model converged, and we did not need to remove any observations, but the estimator rejected some of the samples at each stage. Using a larger neighborhood might allow us to use smaller grid boxes.

We experimented with larger spatial neighborhoods by adding the current period's four diagonal neighbor sites using a single parameter to the model. These sites are the NE, SE, SW and NW neighbor regions, with parameter $\gamma_{0 d}$ (the diagonal term). We have the same model described by Eq. 1, with Eq. 2 changed to

$$
\begin{aligned}
& \log \left(\lambda_{t, i j}\right)= \\
& \quad \mathrm{C}_{i j}(t)+ \\
& \gamma_{0 h}\left(H_{t, i+1, j}+H_{t, i-1, j}\right)+ \\
& \quad \gamma_{0 v}\left(H_{t, i, j+1}+H_{t, i, j-1}\right)+ \\
& \quad \gamma_{0 d}\left(H_{t, i+1, j+1}+H_{t, i-1, j+1}+H_{t, i-1, j-1}+H_{t, i+1, j-1}\right)+ \\
& \quad \gamma_{1 c}\left(H_{t-1, i, j}\right)
\end{aligned}
$$

and the local neighborhood of site $(t, i j), h_{\partial(t, i j)}$, is the vector of nine response values.

We run the modified MCML estimator for several iterations, using the previous values of the parameter estimates from the full model in Table 2, and an initial MPLE estimate for $\gamma_{0 d}$ of 0.0 We estimate that $\gamma_{0 d}=0.090, \sigma_{\gamma_{0 d}}=0.021, t=4.3$. Adding the diagonal parameter to the model significantly affects only the estimates for $\gamma_{0 h}$ and $\gamma_{0 v}$ reducing them to 0.319 and 0.209 respectively, while increasing their standard errors to 0.017 and 0.026 , respectively. The correlation matrix of the coupling parameters and the intercept show that while the other terms have correlations less than 0.07 with each other $\gamma_{0 d}$ is significantly correlated. This term is not only significant, but improves the model. If this term is added both the estimated AIC and SBC are reduced by 17.2 and 10.9 respectively. Thus, future models should consider both larger spatial and temporal neighborhoods.

There are concerns about increasing the size of the neighborhood. If the new couplings are positive the estimator may fail to converge. Because we have more site values, the model will required additional boundary values and parameters. One may reduce the number of parameters by using combinations of various canonical parameters. For example, the parameter $\gamma_{0 d}$ is really the sum of two parameters one for the NW-SE diagonal and another for the NE-SW diagonal. 

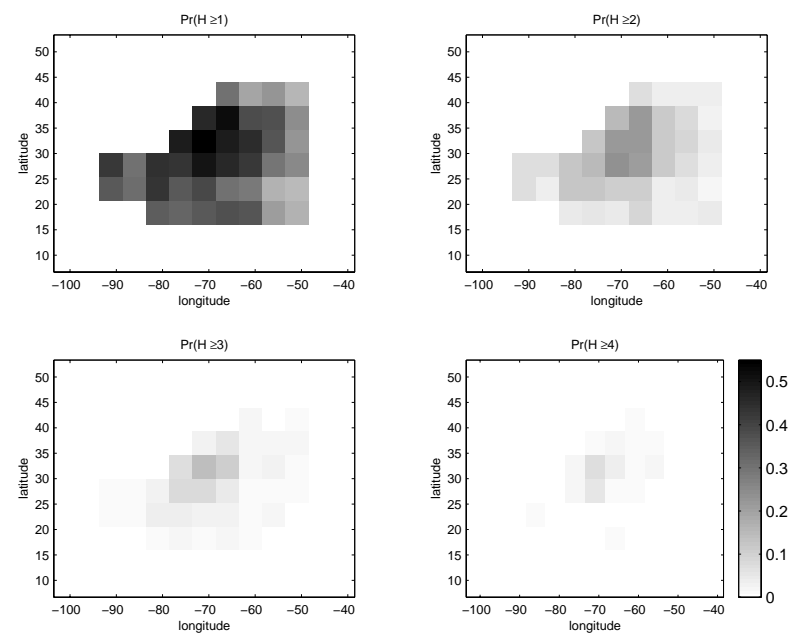

Figure 3: Probabilistic hindcasts of hurricane activity for the 1994 North Atlantic hurricane season. Gray levels indicate the hindcast probability that a given region will have $H$ or more hurricanes.

\subsection{Hindcast}

The model can be used to forecast hurricane activity when lagged values of the covariates are included. At each stage of the MCML estimator, we generate samples from the distribution of $\boldsymbol{X}_{t}$ conditioned on $\left\{\boldsymbol{X}_{s}: s<t\right\}$ using the observations $\left\{\boldsymbol{X}_{t-1}, \ldots \boldsymbol{X}_{t-p}\right\}$, the covariates at time $t$, $z_{k}(t), \quad k=1 \ldots P$ the model parameters and estimated boundary values. For our application we used the full model parameters given in Table 2, so as to have the smallest prediction error. Also, we estimate the boundary values using the mean for each cell.

As a single test case, we generate 103 sample forecasts of hurricane activity for 1994 in each $6^{\circ}$ by $6^{\circ}$ region of grid $S$ using the 1994 values of the five global covariates. Hurricane activity during 1994, which was below the long-term average, is not included at any phase of model development. The spatial distribution and intensity of hindcast values are plotted in Fig. 3.

Results are summarized using boxplots of the predicted probability as a function of actual occurrence as shown in Fig. 4. Seven grid boxes were affected by hurricanes during 1994. The average hindcast probability over those sites is 0.66 with a median value of 0.59 . This compares with an average and median probability of 0.43 and 0.41 , respectively for the 33 sites not affected by hurricanes during 1994. This represents an RMSE error of .2086 as compared to 0.2322 for climatology, a $10 \%$ improvement.

Overall, this single case study supports the contention that the TPSTAR model, or similar spatial count models, might be useful tools in predicting re-

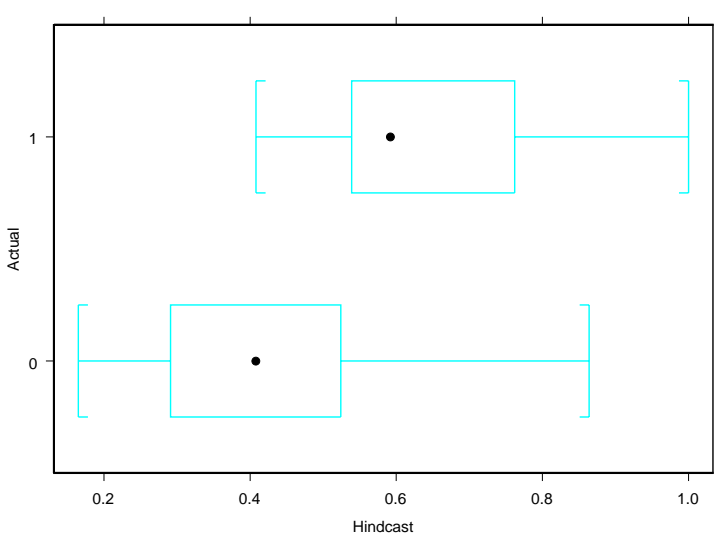

Figure 4: Hindcast probability versus actual occurrence for 1994. The probabilities are generated from the TPSTAR model.

gional hurricane activity over the North Atlantic basin.

\section{SUMMARY AND CONCLUSIONS}

We introduce and apply a space-time count process model to North Atlantic hurricane activity. The model uses the best-track data consisting of historical hurricane positions and intensities together with climate variables to determine local space-time coefficients of a truncated Poisson process. The model, referred to as a truncated Poisson space-time autoregressive (or TPSTAR) model, is motivated by first examining a time-series model for the entire domain. Then a Poisson generalized linear model is considered that uses grids boxes within the domain and adds offset factors for latitude and longitude. A natural extension is then made that includes instantaneous local and autoregressive coupling between the grids. A final version of the model is found by backward selection of the predictors based on values of SBC and AIC. A single hindcast is performed on the 1994 hurricane season using a model having five nearest neighbors and statistically significant couplings. The parameters in the TPSTAR model are estimated using MPLE. The model showed promise as a potential forecast tool.

Several conclusions concerning the application of the TPSTAR model to seasonal North Atlantic hurricane activity are reached:

- Seasonal hurricane activity over the North Atlantic basin can be modeled as a space-time Poisson process.

- There appears to be some temporal autocorrelation in hurricane activity between seasons. 
- Dividing the region into grid boxes and adding coupling increases the significance of the global predictors.

- Increasing the neighborhood size can improve the model at the cost of estimating additional parameters and boundary values.

- From the MCML estimates $t$-tests can be performed to determine if the instantaneous couplings are significant. If they are not we can use the simpler Poisson GLM model.

- Grid size is important to the model formulation. The spatial parameters $\gamma_{0 v}$ and $\gamma_{0 h}$ do not scale with changing grid size, and the MCML estimator may fail to converge for small grid size.

- A hindcast case study provides no evidence against the hypothesis that the TPSTAR modeling procedure can be useful as a climate prediction tool.

Acknowledgements. The study was partially funded by the National Science Foundation (ATM-0086958) and the Risk Prediction Initiative of the Bermuda Biological Station for Research (RPI-99-001).

\section{REFERENCES}

- Arguez A, Elsner JB. 2001. Trends in U.S. tropical cyclone mortality during the 20th century. The Florida Geographer. in press.

- Bartlett MS. 1968. A further note on nearestneighbor models. Journal of the Royal Statistical Society. 131A: 579-580.

- Besag JE. 1974. Spatial interaction and the statistical analysis of lattice systems. Journal of the Royal Statistical Society. 36B:192-225.

- Brockwell P, Davis A. 1991. Time Series: Theory and Methods, Second Edition. SpringerVerlag: New York.

- Elsner JB, Kara AB. 1999. Hurricanes of the North Atlantic: Climate and Society. Oxford University Press: New York.

- Elsner JB, Bossak BH. 2001. Changes in hurricane landfall probabilities in response to variations in climate. Hurricanes and Typhoons: Past, Present, and Future. Murnane RJ, Liu $\mathrm{Kb}$. Eds. Columbia University Press. in preparation.
- Elsner JB, Kara AB, Owens MA. 1999. Fluctuations in North Atlantic hurricanes. Journal of Climate. 12: 427-437.

- Elsner JB, Lehmiller GS, Kimberlain TB. 1996. Early August forecasts of Atlantic tropical storm activity for the balance of the 1996 season, using Poisson models. Experimental Long-lead Forecast Bulletin. 5(3): 26-28.

- Elsner JB, Niu XF, Tsonis AA. 1998. Multi-year prediction model of North Atlantic hurricane activity. Meteorology and Atmospheric Physics. 68: 42-51.

- Elsner JB, Liu Kb, Kocher B. 2000. Spatial variations in major U.S. hurricane activity: Statistics and a physical mechanism. Journal of Climate. 13: 2293-2305.

- Geyer CM. 1994. On the convergence of Monte Carlo maximum likelihood calculations. Journal of the Royal Statistical Society. 56B: 261274.

- Gilks WR, Richardson S, Spiegelhalter DJ. 1996. Markov Chain Monte Carlo in Practice. Chapman \& Hall: London.

- Gray WM. 1984. Atlantic seasonal hurricane frequency: Part II. Forecasting its variability. Monthly Weather Review. 112: 1669-1683.

- Jagger TH. 2000. "A Truncated Space-Time Autoregressive Model." Ph.D. Thesis. Department of Statistics, Florida State University.

- Jagger TH, Elsner JB, Niu XF. 2001. A dynamic probability model of hurricane winds in coastal counties of the United States. Journal of Applied Meteorology. 40: 853-863.

- Landsea CW, Gray WM. 1992. The strong association between Western Sahel monsoon rainfall and intense Atlantic hurricanes. Journal of Climate. 5: 435-453.

- Lehmiller GS, Kimberlain TB, Elsner JB. 1997. Seasonal prediction models for North Atlantic basin hurricane location. Monthly Weather Review. 125: 1780-1791.

- Neumann CJ, Jarvinen BR, McAdie CJ, Hammer GR. 1999. Tropical Cyclones of the North Atlantic Ocean, 1871-1998. National Oceanic and Atmospheric Administration. 
- Sharpiro LJ. 1987. Month-to-month variability of the Atlantic tropical circulation and its relationship to tropical storm formation. Monthly Weather Review. 115: 2598-2614.

- Whittle P. 1963. Stochastic processes in several dimensions. Bulletin of the International Statistical Institute. 40: Book 2.

- Winkler G. 1995. Image Analysis, Random Fields, and Dynamic Monte Carlo Methods, Springer-Verlag: Berlin.

- Wu H. 1994. "Regression Models for Spatial Binary Data with Application to the Distribution of Plant Species." Ph.D. Thesis. Department of Statistics, Florida State University. 